Check for updates

Cite this: RSC Adv., 2017, 7, 44884

Received 6th July 2017

Accepted 22nd August 2017

DOI: $10.1039 / c 7 r a 07455 g$

rsc.li/rsc-advances

\title{
Unexpected stable stoichiometries and superconductivity of potassium-rich sulfidest+
}

\author{
Ying Li, (D) Xilian Jin, (D)* Tian Cui, (D)* Quan Zhuang, Die Zhang, Xing Meng, Kuo Bao, \\ Bingbing Liu (D) and Qiang Zhou
}

\begin{abstract}
Alkali metal compounds exhibit novel characteristics under pressure, such as antimetallization in $\mathrm{CLi}_{4}$, high superconductivity at $80 \mathrm{~K}$ in highly compressed $\mathrm{Li}_{3} \mathrm{~S}$, and the existence of unexpected stable stoichiometries of sodium chlorides, etc., which have greatly prompted us to explore $K_{x} S$ compounds at pressure. We found several stable structures with a variety of stoichiometries and proposed a phase diagram on the $\mathrm{K}$-rich side first. Chemical rules established at ambient pressure are frequently violated when high pressure is applied, $\mathrm{Na}_{3} \mathrm{Cl}$ and $\mathrm{NaCl}_{3}$ as unusual stoichiometries of sodium chloride have been reported in high-pressure conditions. However, KS, with its counterintuitive chemical formula, has been discovered theoretically even at ambient pressure, and possesses the same stability as $\mathrm{K}_{2} \mathrm{~S}$. The mechanism of superconductivity in $P m \overline{3} m \mathrm{~K}_{3} \mathrm{~S}$ is deeply investigated, comparing with the reported $\mathrm{Pm} \overline{3} \mathrm{~m} \mathrm{Li}{ }_{3} \mathrm{~S}$. The weak electron-phonon coupling mainly contributes to the weak superconductivity in $\mathrm{K}_{3} \mathrm{~S}$, which is fully in contrast to the mechanism of interstitial charge localization which dominates the low $T_{\mathrm{C}}$ in the reported $\mathrm{Li}_{3} \mathrm{~S}$.
\end{abstract}

\section{Introduction}

Recently, alkali metal compounds have revived the interest of the scientific community to explore their novel structures and characteristics upon compression. Different $\mathrm{Na}-\mathrm{Cl}$ compounds such as $\mathrm{Na}_{3} \mathrm{Cl}, \mathrm{Na}_{2} \mathrm{Cl}, \mathrm{Na}_{3} \mathrm{Cl}_{2}, \mathrm{NaCl}_{3}$, and $\mathrm{NaCl}_{7}$ are thermodynamically stable at nonambient pressure conditions which contrasts the familiar rules of chemistry. ${ }^{1,2}$ The compound of helium and sodium, $\mathrm{Na}_{2} \mathrm{He}$, reported recently has changed the hitherto bare field of helium chemistry, providing new twists to the chemistry of noble gases. ${ }^{3}$ Antimetallization is predicted in the dense lithium-rich compound $\mathrm{CLi}_{4}$ (ref. 4 ) which means that this phenomenon is expected in not only alkali metals $\mathrm{Li}^{5-7}$ and $\mathrm{Na}^{8}$ but also multiple compounds. Interestingly, superconductivity is predicted in alkali metal compounds such as a $\mathrm{Li}-\mathrm{S}$ system, and $T_{\mathrm{c}}$ has risen to $80 \mathrm{~K}$ in highly compressed $\mathrm{Li}_{3} \mathrm{~S} .{ }^{9}$

Alkali metal sulfides are characterized by their high ionic conductivity and large band gap. They appear to be promising candidates for technological applications in solid state batteries, fuel cells and solid state gas-detectors. ${ }^{\mathbf{1 0 1 1}}$ The properties of alkali metal sulfides of $\mathrm{X}_{2} \mathrm{~S}(\mathrm{X}=\mathrm{Li}, \mathrm{Na}, \mathrm{K}$, or $\mathrm{Rb})$ have been explored thoroughly, including phase sequence, elastic properties, electronic structure, optical properties, etc. ${ }^{12-20}$ The

College of Physics, State Key Laboratory of Superhard Materials, Jilin University, Changchun, 130012, People's Republic of China. E-mail: jinxilian@jlu.edu.cn; cuitian@jlu.edu.cn

$\dagger$ Dedicated to Prof. Guangtian Zou on the occasion of his $80^{\text {th }}$ birthday.

\$ Electronic supplementary information (ESI) available. See DOI: $10.1039 / \mathrm{c} 7 \mathrm{ra} 07455 \mathrm{~g}$ conventional superconductivity in the phase diagram of the Li$\mathrm{S}$ system has been explored by $a b$ initio methods for crystal structure prediction and linear response calculations for electron-phonon coupling. ${ }^{9}$ Most of these phases are metallic, but they exhibit no or low- $T_{\mathrm{c}}$ superconductivity, except for the $F m \overline{3} m$ space group of $\mathrm{Li}_{3} \mathrm{~S}$ with a $T_{\mathrm{c}}$ of $80 \mathrm{~K}$ at $500 \mathrm{GPa}$. In contrast to the low- $T_{\mathrm{c}}$ of $P m \overline{3} m \mathrm{Li}_{3} \mathrm{~S}$, it is concluded that interstitial charge localization due to avoiding core overlap can be a fundamental limiting factor for conventional superconductivity.

In this work, various stoichiometric structures of $\mathrm{K}_{x} \mathrm{~S}(x=1-$ 4) systems are widely investigated up to $100 \mathrm{GPa}$. We found six thermodynamically stable structures with the stoichiometries of $\mathrm{KS}, \mathrm{K}_{3} \mathrm{~S}$ and $\mathrm{K}_{4} \mathrm{~S}$ under these conditions and proposed a phase diagram on the K-rich side first. KS, the totally counterintuitive chemical formula with extraordinary bonding and electronic properties, is predicted to be stable at atmospheric pressure in $\mathrm{K}_{x} \mathrm{~S}$ compounds. The mechanism of superconductivity in $P m \overline{3} m \mathrm{~K}_{3} \mathrm{~S}$ is deeply investigated and compared with the reported $P m \overline{3} m \mathrm{Li}_{3} \mathrm{~S}$. The low $T_{\mathrm{c}}$ is attributed to a weak interaction of electron-phonon coupling in the $\mathrm{K}_{3} \mathrm{~S}$ compound instead of interstitial charge localization like in the reported $\mathrm{Li}_{3} \mathrm{~S}$ compound. ${ }^{9}$

\section{Computational methods}

The structural prediction for the $\mathrm{K}_{x} \mathrm{~S}$ compounds at different pressures was performed by the ELocR code. ${ }^{21}$ The high accuracy structural relaxations and electronic localization functions (ELF) were performed using density functional theory with the 
Vienna ab initio simulation package (VASP). ${ }^{22}$ The PerdewBurke-Ernzerhof (PBE) generalized gradient approximation $(\mathrm{GGA})^{23}$ was selected for the exchange-correlation function. The partial augmented wave (PAW) ${ }^{24}$ method was adopted with the PAW potentials where $\mathrm{s}^{2} \mathrm{p}^{4}$ and $3 \mathrm{~s}^{3} \mathrm{p}^{4} \mathrm{~s}$ are treated as valence electrons. Brillouin zone sampling used a grid spacing of $2 \pi \times$ $0.03 \AA^{-1}$ and the plane-wave cutoff energy was $600 \mathrm{eV}$.

The electronic projected density of states and the electronic band structure were calculated by the CASTEP ${ }^{25}$ code with a cutoff energy of $720 \mathrm{eV}$, norm-conserving pseudopotentials, GGA-PBE exchange-correlation functional, and Brillouin zone sampling grid with a spacing of $2 \pi \times 0.04 \AA^{-1}$. The dynamic properties, three-dimensional Fermi surfaces and electronphonon coupling calculations were studied in the QUANTUMESPRESSO package. ${ }^{26}$ The Troullier-Martins-type normconserving pseudopotentials were used, and convergence tests provided a suitable value of 60 Ry for the kinetic energy cutoff. The q-point meshes in the first Brillouin zone of $8 \times 8 \times 8$ for $P m \overline{3} m\left(\mathrm{Li}_{3} \mathrm{~S}\right)$ and $4 \times 4 \times 4$ for $P m \overline{3} m\left(\mathrm{~K}_{3} \mathrm{~S}\right)$ were used for phonon and electron-phonon calculations.

\section{Results and discussion}

We have systematically studied the chemical stabilities of various $K_{x} S(x=1-4)$ compounds by calculating the enthalpies of formation up to $100 \mathrm{GPa}$. The thermodynamical stability of different compounds with respect to elemental $\mathrm{K}$ and $\mathrm{S}$ solids at each pressure is calculated using the equation below:

$$
\Delta H\left(\mathrm{~K}_{x} \mathrm{~S}\right)=\left[\left[H\left(\mathrm{~K}_{x} \mathrm{~S}\right)-x H(\mathrm{~K})-H(\mathrm{~S})\right](x+1)\right]
$$

where $\Delta H$ is the enthalpy of formation per atom and $H$ is the calculated enthalpy per chemical unit for each compound. All possible structures with lower enthalpies are provided in the convex hull, as shown in Fig. 1 and S1.\$

According to the hull data, a compound lying on the convex hull is thermodynamically stable with respect to decomposition

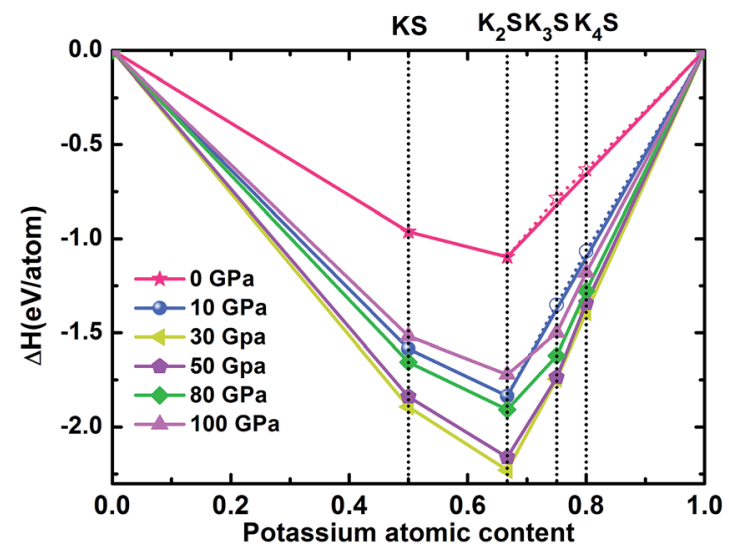

Fig. 1 Enthalpy difference curves of $K_{x} S$ with respect to $K$ and $S$ at selected pressures. The $K_{x} S$ structures on the convex hull (solid lines) are thermodynamically stable relative to decomposition into other $K_{x} S$ compounds and elements, whereas those located above the convex hull (dashed lines) are unstable or metastable.

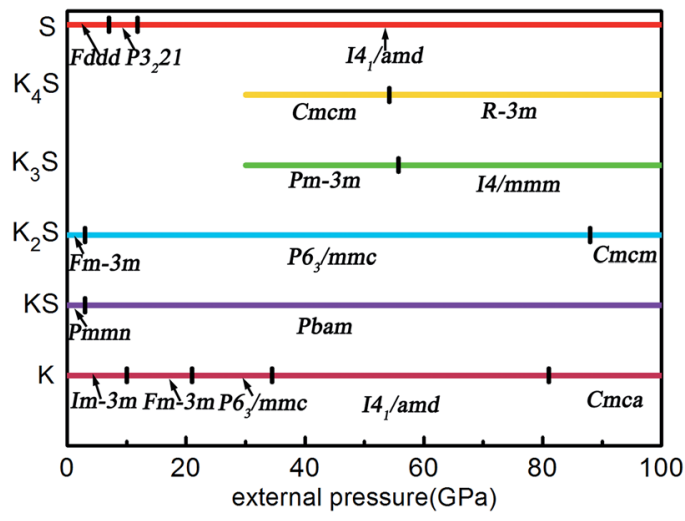

Fig. 2 The phase diagram of the $K_{x} S$ system in the stable pressure range.

into other $\mathrm{K}-\mathrm{S}$ compounds or elemental $\mathrm{K}$ and $\mathrm{S}$ solids, and thus it is experimentally synthesizable. However, those located above the convex hull indicated by dashed lines are either unstable or metastable. In order to obtain clear information for the structures at ambient conditions and $10 \mathrm{GPa}$, the independent convex hull is shown in Fig. S1.\$ Moreover, the phase diagram for the $\mathrm{K}_{x} \mathrm{~S}$ crystals is presented in Fig. 2.

Under ambient conditions, the stable phase of KS crystallizes in the orthorhombic structure of Pmmn and remains stable until 3 GPa as shown in Fig. 3(a). There are three nonequivalent atoms, and the nearest distance between $\mathrm{K}$ and $\mathrm{S}$ is $3.16 \AA$. $\mathrm{K}$ atoms occupy the crystallographic $2 \mathrm{~b}$ position with $\mathrm{mm} 2$ symmetry on the top and bottom sides, and the other nonequivalent $\mathrm{K}$ atoms occupy the crystallographic 2a position with $\mathrm{mm} 2$ symmetry in the middle of the crystal. S atoms occupy the crystallographic $4 \mathrm{f}$ position with.$m$. symmetry. Elevating the pressure above $3 \mathrm{GPa}$, another orthorhombic phase with Pbam symmetry emerges which is stable until $100 \mathrm{GPa}$, as depicted in Fig. 3(b). There are two nonequivalent atoms in the crystal lattice, and they consist of one KS unit. $\mathrm{K}$ atoms occupy the crystallographic $4 \mathrm{~h}$ position with ..m symmetry, and $\mathrm{S}$ atoms occupy the crystallographic $4 \mathrm{~g}$ position with ..m symmetry. The nearest distance between $\mathrm{K}$ and $\mathrm{S}$ is $2.558 \AA$. As for $\mathrm{K}_{3} \mathrm{~S}$ and $\mathrm{K}_{4} \mathrm{~S}$, they are thermodynamically metastable under normal conditions until $30 \mathrm{GPa}$. With increasing pressure, $P m \overline{3} \mathrm{~m}$, the facecentered cubic phase, is energetically preferred from $30 \mathrm{GPa}$
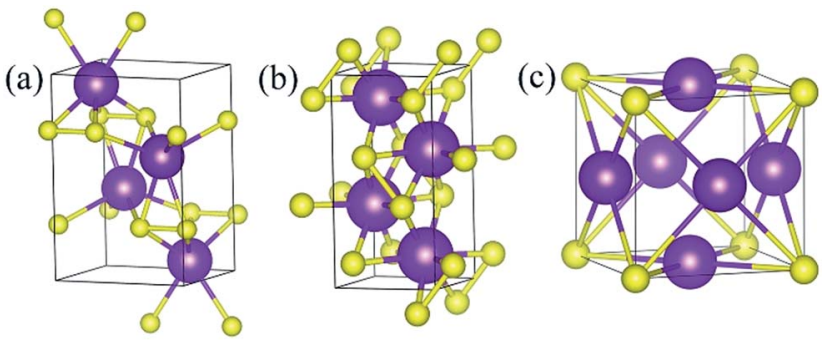

Fig. 3 Stable structures of $\mathrm{K}_{x} \mathrm{~S}$ : (a) Pmmn KS at ambient pressure, (b) Pbam KS at $100 \mathrm{GPa}$, and (c) $P m 3 m \mathrm{~K}_{3} \mathrm{~S}$ at $50 \mathrm{GPa}$. The purple and yellow atoms are $\mathrm{K}$ and $\mathrm{S}$, respectively. 


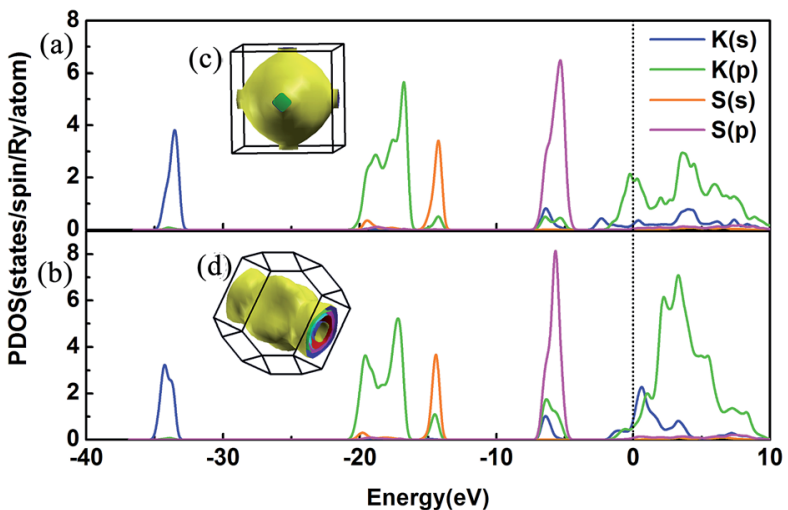

Fig. 4 PDOS and the three-dimensional Fermi surfaces of $\mathrm{K}_{3} \mathrm{~S}$. (a) PDOS of $P m 3 \mathrm{~m}$ at $50 \mathrm{GPa}$, (b) PDOS of $14 / \mathrm{mmm}$ at $100 \mathrm{GPa}$, (c) the three-dimensional Fermi surfaces of $P m 3 m$ at $50 \mathrm{GPa}$, and (d) the three-dimensional Fermi surfaces of $14 / \mathrm{mmm}$ at $100 \mathrm{GPa}$. The black vertical dashed line indicates the Fermi energy.

to $55.7 \mathrm{GPa}$. The layer stacking sequence in every axis can be denoted by the repeated $\mathrm{AB}$ stacking. $\mathrm{K}$ atoms occupy the crystallographic $3 \mathrm{c}$ position with $4 / \mathrm{mm} . \mathrm{m}$ symmetry sitting at the center of each cube face. $\mathrm{S}$ atoms occupy the crystallographic 1a position with $m \overline{3} m$ symmetry around the cube corners as shown in Fig. 3(c). Each $\mathrm{K}$ atom is surrounded by the four nearest $\mathrm{S}$ atoms and the distance is $2.779 \AA$. The calculated structural parameters and Wyckoff positions for each phase are summarized in Table S1. The mechanical property is one of the basic criteria when considering the structure stability. According to the mechanical stability criteria, the crystal deformation energy is positive which means the determinants of the elastic constants matrix $C_{i j}$ should be positive. ${ }^{27}$ It is noteworthy that negative values are not prohibited for $C_{i j}{ }^{28}$ To evaluate the mechanical stability of Pmmn, Pbam, $P m \overline{3} \mathrm{~m}, I 4 / \mathrm{mmm}, \mathrm{Cmcm}$ and $R \overline{3} m$, the elastic constants were calculated and are listed in Table S2. They all satisfy the Born-Huang criterion, ${ }^{29}$ representing the stability of the mechanical property.

$\mathrm{KS}$ and $\mathrm{K}_{2} \mathrm{~S}$ are thermodynamically stable compounds throughout the entire pressure range we explored. At ambient pressure, $\mathrm{K}_{2} \mathrm{~S}$ is the familiar stoichiometry in the $\mathrm{K}_{x} \mathrm{~S}$ system. We have predicted its phase sequence successfully by the ELocR $^{21}$ code, and account for the experimental results effectively. ${ }^{20}$ Pressure can efficiently modify the compositional landscape, leading to materials with unprecedented stoichiometries that would not be expected from conventional wisdom based on chemical rules at ambient pressure. For instance, at ambient conditions, sodium chloride $(\mathrm{NaCl})$ is the archetypical ionic compound in chemistry textbooks. Under pressure, different $\mathrm{Na}-\mathrm{Cl}$ compounds such as $\mathrm{Na}_{3} \mathrm{Cl}, \mathrm{Na}_{2} \mathrm{Cl}, \mathrm{Na}_{3} \mathrm{Cl}_{2}$, $\mathrm{NaCl}_{3}$, and $\mathrm{NaCl}_{7}$, which are clearly against our conventional wisdom, become thermodynamically stable., ${ }^{\mathbf{1 , 2}}$ Some other examples are seen in $\mathrm{Li}-\mathrm{B},{ }^{30,31} \mathrm{Ca}-\mathrm{H},{ }^{32}$ and $\mathrm{Si}-\mathrm{C}^{33}$ systems at high pressure conditions. Interestingly, the unusual stoichiometry (1:1) KS compound is even stable thermodynamically and mechanically at ambient conditions. In order to judge its dynamical stability, the phonon band structure and partial phonon density of states (PHDOS) were calculated as shown in Fig. S2.t The absence of imaginary frequency modes in the entire Brillouin zone indicates the dynamic stability of Pmmn (KS). Hence, KS, the totally counterintuitive chemical formula, may exist in nature theoretically.

We have discussed the mechanical and thermodynamical stability of the proposed phases and confirmed that they are all stable at the relevant pressure range. To understand the electronic properties of the various $\mathrm{K}_{x} \mathrm{~S}$ compounds, the electronic band structures, partial density of states (PDOS) and threedimensional Fermi surfaces were calculated as depicted in Fig. 4 and S3. From the band structures we found that all the stable phases at the different pressures are metallic due to several bands crossing the Fermi level, except for Pmmn and Pbam. At ambient pressure, the band gap in Pmmn is $1.432 \mathrm{eV}$, which reveals its nonmetallic character. Elevating the pressure above $3 \mathrm{GPa}$, the Pbam structure appears with nonmetallic features until $100 \mathrm{GPa}$. For $\mathrm{K}_{3} \mathrm{~S}$, from the partial density of states (PDOS) in Fig. 4(a) and (b), the metallic character of $P m \overline{3} m$ and $I 4 / \mathrm{mmm}$ is confirmed by the high level of total electronic density distribution at the Fermi level. The majority of the occupied states come from the $\mathrm{K}(\mathrm{p})$ state, whereas the contribution from
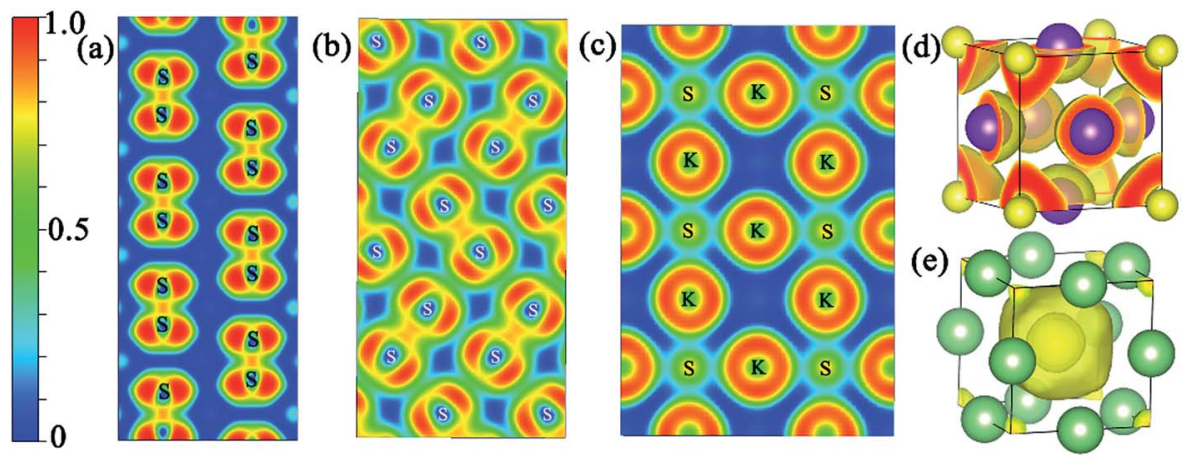

Fig. 5 The electron localization function of $\mathrm{KS}, \mathrm{K}_{3} \mathrm{~S}$ and $\mathrm{Li}_{3} \mathrm{~S}$. (a) Pmmn 2D ELF slice along the (011) plane at ambient pressure; (b) Pbam 2D ELF slice along the (001) plane at $100 \mathrm{GPa}$; (c) Pm3m K3 S 2D ELF slice along the (001) plane at $50 \mathrm{GPa}$; (d) Pm3m K3 $3 \mathrm{D}$ ELF map with the ELF values of 0.65 at $50 \mathrm{GPa}$; (e) $\mathrm{Pm} \overline{3} \mathrm{~m} \mathrm{Li}{ }_{3} \mathrm{~S}$ 3D ELF map with the ELF values of 0.65 at $500 \mathrm{GPa}$. Pmm $\mathrm{Li}_{3} \mathrm{~S}$ 3D ELF map with the ELF values of 0.65 at $500 \mathrm{GPa}$. 


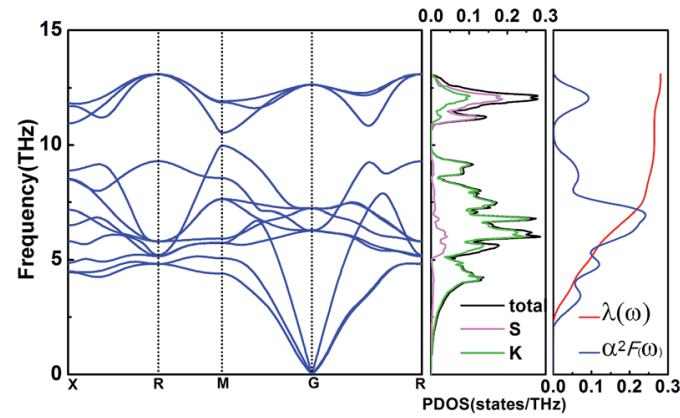

Fig. 6 Phonon dispersion curves, partial phonon density of states (PDOS), Eliashberg spectral function $\alpha^{2} F(\omega)$, and the EPC $\lambda$ for Pm3m $\left(\mathrm{K}_{3} \mathrm{~S}\right)$ at $50 \mathrm{GPa}$.

$\mathrm{K}(\mathrm{s}), \mathrm{S}(\mathrm{s})$ and $\mathrm{S}(\mathrm{p})$ to the states around the Fermi level is quite small in $\mathrm{K}_{3} \mathrm{~S}$. This complex electronic band structure near the Fermi energy brings the rich and multiple Fermi surface feature, as displayed in Fig. 4(c) and (d).

In addition, the electron localization function (ELF) was calculated to analyze the nature of the chemical bonding and the unusual distribution of the valence electron localization function in the proposed structures at the relevant pressure range. It is a ratio from 0 to 1 , and the values equal 1.0 and 0.5 , reflecting the extremely strong localization and homogeneous electron distribution, respectively. ${ }^{34,35}$ Fig. S4 $\$$ shows the isosurface value of 0.65 for the proposed structures. The valence electron localization around interstitial regions is found in Pmmn (KS), Pbam (KS), I4/mmm $\left(\mathrm{K}_{3} \mathrm{~S}\right), C m c m\left(\mathrm{~K}_{4} \mathrm{~S}\right)$, and $R \overline{3} m$ $\left(\mathrm{K}_{4} \mathrm{~S}\right)$, and is not found in $P m \overline{3} m\left(\mathrm{~K}_{3} \mathrm{~S}\right)$. Fig. 5(a) and (b) show the two dimensional electron location function (2D ELF) of Pmmn (KS) and Pbam (KS). The values around the positions of the $\mathrm{S}_{2}$ units are close to 0.75 , revealing the covalent bond character. From the 2D ELF of $P m \overline{3} m\left(\mathrm{~K}_{3} \mathrm{~S}\right)$ displayed in Fig. 5(c), we can see that the conductivity comes from connected regions where the value of ELF is about 0.5 between the K and S atoms. The ELF of $P m \overline{3} m\left(\mathrm{Li}_{3} \mathrm{~S}\right)$ at $500 \mathrm{GPa}$ is calculated to compare with $\mathrm{K}_{3} \mathrm{~S}$ as shown in Fig. 5(e). Obviously, charges accumulate in the open interstitial regions in $\mathrm{Li}_{3} \mathrm{~S}$. However, this phenomenon is not observed in $P m \overline{3} m \mathrm{~K}_{3} \mathrm{~S}$ as displayed in Fig. 5(d).

To explore the potential superconductivity of $P m \overline{3} m \mathrm{~K}_{3} \mathrm{~S}$, the electron-phonon coupling (EPC) strength $\lambda$ and logarithmic average phonon frequency $\omega_{\log }$ have been investigated at $50 \mathrm{GPa}$ by QUANTUM-ESPRESSO. ${ }^{26}$ The Eliashberg phonon spectral function $\alpha^{2} F(\omega)$ can be obtained as below. It is described as:

$$
\alpha^{2} F(\omega)=\frac{1}{2 \pi N\left(\varepsilon_{\mathrm{F}}\right)} \sum_{q v} \frac{\gamma_{q v}}{\omega_{q v}} \delta\left(\omega-\omega_{q v}\right)
$$

hereinto the line width:

$$
\gamma_{q v}=\lambda_{q v} \pi N\left(\varepsilon_{\mathrm{F}}\right) \omega_{q v}{ }^{2}
$$

so, we can obtain $\alpha^{2} F(\omega)$ :

$$
\alpha^{2} F(\omega)=\frac{1}{2} \sum_{q v} \lambda_{q v} \omega_{q v} \delta\left(\omega-\omega_{q v}\right)
$$

In this work, $\alpha^{2} F(\omega)$ is obtained by the integration of $\omega_{q v}$ in the whole Brillouin zone. The heavier $\mathrm{K}$ atom from the lowfrequency vibrational modes below about $8 \mathrm{THz}$ contributes more to the total $\lambda$ as shown in Fig. 6. Phonon-mediated superconductors are accurately described by the Migdal-Eliashberg theory, ${ }^{36,37}$ and $T_{\mathrm{c}}$ can be estimated by the Allen-Dynes formula: ${ }^{38}$

$$
T_{\mathrm{c}}=\frac{\omega_{\log }}{1.2} \exp \left[\frac{-1.04(1+\lambda)}{\lambda-\mu^{*}(1+0.62 \lambda)}\right]
$$

which has been found to be highly accurate for many materials with $\lambda<1.5$. We chose the better recommended values of the Coulomb pseudopotential $\mu^{*}(0.1-0.13){ }^{39}$ and the superconducting transition temperature $T_{\mathrm{c}}$ was estimated to nearly $0 \mathrm{~K}$ at $50 \mathrm{GPa}$. A recent theoretical study reported that $\mathrm{Li}_{3} \mathrm{~S}$ exhibits low- $T_{\mathrm{c}}(0 \mathrm{~K})$ in the $P m \overline{3} m$ phase and high- $T_{\mathrm{c}}(80 \mathrm{~K})$ in the $F m \overline{3} m$ phase, and it attributed the difference to interstitial charge localization, i.e., charge localizing in the interstitial region is a limiting factor to conventional superconductivity. ${ }^{9}$ $\mathrm{K}_{3} \mathrm{~S}$ has the same stoichiometry, space group and $T_{\mathrm{c}}$ as $\mathrm{Li}_{3} \mathrm{~S}$, however, the mechanism of superconductivity is totally different. We attribute the low $T_{\mathrm{c}}$ to weak electron-phonon coupling. As expected, in $\mathrm{K}_{3} \mathrm{~S}$ and $\mathrm{Li}_{3} \mathrm{~S}, T_{\mathrm{c}}$ is dominated by $\lambda$. Then the variation of $\lambda$ is analysed using the rigid-muffin-tin (RMT) theory of Gaspari and Gyorffy. ${ }^{40}$ McMillan's strong coupling theory defines an electron-phonon coupling constant by

$$
\lambda=\frac{N\left(\varepsilon_{\mathrm{F}}\right)\left\langle I^{2}\right\rangle}{M\left\langle\omega^{2}\right\rangle}=\eta N\left(\varepsilon_{\mathrm{F}}\right)
$$

where $\eta=\frac{\left\langle I^{2}\right\rangle}{M\left\langle\omega^{2}\right\rangle} . N\left(\varepsilon_{\mathrm{F}}\right)$ is the density of states (DOS) at the Fermi level, $M$ is the atomic mass, $\left\langle\omega^{2}\right\rangle$ is a weighted average of

Table 1 The calculated electronic density of states at the Fermi level $N\left(\varepsilon_{F}\right)$ (states/spin/Ry/atom), the logarithmic average phonon frequency $\omega_{\text {log }}$ $(\mathrm{K})$, the average phonon frequency $\left\langle\omega^{2}\right\rangle^{1 / 2}(\mathrm{THz})$, the average over the Fermi surface of the electron-phonon matrix element $\left\langle\mathrm{I}^{2}\right\rangle\left(\mathrm{eV} \AA^{-1}\right)$, ${ }^{2}$ the electron-phonon coupling parameters $\lambda$, and the superconducting critical temperatures $T_{c}(\mathrm{~K})$ of $\mathrm{K}_{3} \mathrm{~S}$ and $\mathrm{Li}_{3} \mathrm{~S}$ at $50 \mathrm{GPa}$ and $500 \mathrm{GPa}$, respectively

\begin{tabular}{llllllllrr}
\hline & & $P$ & & & & & & \\
& Phase & $(\mathrm{GPa})$ & $T_{\mathrm{c}}$ & $\omega_{\log }$ & $\lambda$ & $N\left(\varepsilon_{\mathrm{F}}\right)$ & $\eta$ & $\left\langle\omega^{2}\right\rangle^{1 / 2}$ & $\left\langle I^{2}\right\rangle$ \\
\hline Present work & $P m \overline{3} m\left(\mathrm{~K}_{3} \mathrm{~S}\right)$ & 50 & 0.069 & 285.569 & 0.280 & 2.107 & 0.133 & 40.698 & 2.884 \\
Present work & $P m \overline{3} m\left(\mathrm{Li}_{3} \mathrm{~S}\right)$ & 500 & 0.044 & 814.923 & 0.250 & 0.668 & 0.375 & 141.074 & 25.120 \\
Other calculations & $P m \overline{3} m\left(\mathrm{Li}_{3} \mathrm{~S}\right)^{9}$ & 500 & 0 & 702 & 0.25 & 0.67 & 0.37 & &
\end{tabular}


the square of the phonon frequency, and $\left\langle I^{2}\right\rangle$ is an average over the Fermi surface of the electron-phonon matrix element. From Table $1, \eta$ plays an important role in $\lambda$ which further influenced the low $T_{\mathrm{c}}$ in $\mathrm{Li}_{3} \mathrm{~S}$ and $\mathrm{K}_{3} \mathrm{~S}$. With regard to $\eta$, it is believed that $\eta$ is a factor about the lattice and its value is influenced by interstitial charge localization in $\mathrm{Li}_{3} \mathrm{~S},{ }^{9}$ i.e. charge localizing in the interstitial region led to a smaller $\eta$. The value of $\eta$ in $\mathrm{K}_{3} \mathrm{~S}$ is smaller than that in $\mathrm{Li}_{3} \mathrm{~S}$, nevertheless, there is no charge localizing in the interstitial region as shown in Fig. 5(c). We attribute the small $\eta$ to the electron-ion matrix element $\left\langle I^{2}\right\rangle$. In conclusion, the low $T_{\mathrm{c}}$ in $\mathrm{K}_{3} \mathrm{~S}$ is mainly influenced by electronphonon coupling which is fully in contrast to the mechanism of interstitial charge localization which dominates the low $T_{\mathrm{c}}$ in the reported $\mathrm{Li}_{3} \mathrm{~S} .^{9}$

\section{Conclusions}

In conclusion, a high-pressure phase diagram of K-rich $\mathrm{K}_{x} \mathrm{~S}$ systems was built from structure prediction simulations and first-principles calculations. KS, the totally counterintuitive chemical formula with extraordinary bonding and electronic properties, is predicted to be stable at atmospheric pressure. All of the $\mathrm{S}$ atoms of KS are in the form of $\mathrm{S}_{2}$ units with covalent bond character from the analyses of the electronic localization function. Further electron-phonon coupling calculations revealed that the $T_{\mathrm{c}}$ of $P m \overline{3} m\left(\mathrm{~K}_{3} \mathrm{~S}\right)$ is approximately near to $0 \mathrm{~K}$ at $50 \mathrm{GPa}$. The mechanism of superconductivity in $P m \overline{3} m \mathrm{~K}_{3} \mathrm{~S}$ was deeply investigated and compared with the reported $P m \overline{3} m$ $\mathrm{Li}_{3} \mathrm{~S}$. We attribute the low $T_{\mathrm{c}}$ to weak electron-phonon coupling in the $\mathrm{K}_{3} \mathrm{~S}$ compound.

\section{Conflicts of interest}

There are no conflicts to declare.

\section{Acknowledgements}

This work was supported by the National Natural Science Foundation of China (No. 51632002, 51572108, 11634004, 11174102, 11774119), the Program for Changjiang Scholars and Innovative Research Team in University (No. IRT_15R23), the 111 Project (No. B12011), the National Fund for Fostering Talents of Basic Science (No. J1103202), and the Development Program of Science and Technology of Jilin Province, China (No. 20150312002ZG). Parts of the calculations were performed in the High Performance Computing Center (HPCC) of Jilin University.

\section{References}

1 W. Zhang, A. R. Oganov, A. F. Goncharov, et al., Science, 2013, 342, 1502-1505.

2 J. I. Insa, Science, 2013, 342, 1459-1460.

3 X. Dong, A. R. Oganov, A. F. Goncharov, et al., Nat. Chem., 2017, 9, 440-445.
4 X. Jin, X. J. Chen, T. Cui, H. K. Mao, H. Zhang, Q. Zhuang, K. Bao, D. Zhou, B. Liu, Q. Zhou and Z. He, Proc. Natl. Acad. Sci. U. S. A., 2016, 113, 2366-2369.

5 J. B. Neaton and N. W. Ashcroft, Nature, 1999, 400, 141-144.

6 T. Matsuoka and K. Shimizu, Nature, 2009, 458, 186-189.

7 Y. Yao, S. T. John and D. D. Klug, Phys. Rev. Lett., 2009, 102, 115503.

8 Y. Ma, M. Eremets, A. R. Oganov, et al., Nature, 2009, 458, 182-185.

9 C. Kokail, C. Heil and L. Boeri, Phys. Rev. B, 2016, 94, 060502.

10 P. G. Bruce, S. A. Freunberger, L. J. Hardwick and J. Tarascon, Nat. Mater., 2012, 11, 19-29.

11 H. Momida, T. Yamashita and T. Oguchi, J. Phys. Soc. Jpn., 2014, 83, 124713.

12 H. Khachai, R. Khenata and A. Bouhemadou, Solid State Commun., 2008, 147, 178-182.

13 R. D. Eithiraj, G. Jaiganesh and G. Kalpana, Phys. Status Solidi A, 2007, 244, 1337-1346.

14 J. C. Schön, Ž. Čančarević and M. Jansen, J. Chem. Phys., 2004, 121, 2289.

15 H. Khachai, R. Khenata and A. Bouhemadou, J. Phys., 2009, 21, 095404.

16 E. Zintl, A. Harder and B. Dauth, Z. Elektrochem. Angew. Phys. Chem., 1934, 40, 588-593.

17 A. Grzechnik, A. Vagas, K. Syassen, L. Loa, M. Hanfland and M. Jansen, J. Solid State Chem., 2000, 154, 603-611.

18 A. Vegas, A. Grzechnik, K. Syassen, L. Loa, M. Hanfland and M. Jansen, Acta Crystallogr., Sect. B: Struct. Sci., 2001, 57, 151-156.

19 D. Santamaria-Perez, A. Vegas, C. Muehle and M. Jansen, Acta Crystallogr., Sect. B: Struct. Sci., 2011, 67, 109-115.

20 Y. Li, X. Jin, T. Cui, Q. Zhuang, Q. Lv, G. Wu, X. Meng, K. Bao, B. Liu and Q. Zhou, RSC Adv., 2017, 7, 7424-7430.

21 The code for crystal structural prediction and analysis is based on the evolutional local random computational method, and is developed by our group. Some details have been provided in the ESI.t.

22 G. Kresse and J. Furthmüller, Phys. Rev. B: Condens. Matter Mater. Phys., 1996, 54, 11169-11186.

23 J. P. Perdew, K. Burke and M. Ernzerhof, Phys. Rev. Lett., 1997, 77, 3865-3868.

24 P. E. Blöchl, Phys. Rev. B: Condens. Matter Mater. Phys., 1994, 50, 17953-17979.

25 S. J. Clark, M. D. Segall and C. J. Pickard, Z. Kristallogr. Cryst. Mater., 2005, 220, 567-570.

26 P. Giannozzi, S. Baroni, N. Bonini, M. Calandra, R. Car, C. Cavazzoni, D. Ceresoli, G. L. Chiarotti, M. Cococcioni, I. Dabo, A. Dal Corso, S. de Gironcoli, S. Fabris, G. Fratesi, R. Gebauer, U. Gerstmann, C. Gougoussis, A. Kokalj, M. Lazzeri, L. Martin-Samos, N. Marzari, F. Mauri, R. Mazzarello, S. Paolini, A. Pasquarello, L. Paulatto, C. Sbraccia, S. Scandolo, G. Sclauzero, A. P. Seitsonen, A. Smogunov, P. Umari and R. M. Wentzcovitch, J. Phys.: Condens. Matter, 2009, 21, 395502.

27 J. F. Nye, Physical properties of crystals: their representation by tensors and matrices, Oxford Univ. Press, Oxford, 1985, pp. 142-143. 
28 J. P. Perdew and K. Burke, Phys. Rev. Lett., 1997, 77, 38653868.

29 Z. Wu, E. Zhao and H. Xiang, Phys. Rev. B: Condens. Matter Mater. Phys., 2007, 76, 054115.

30 F. Peng, M. Miao, H. Wang, Q. Li and Y. Ma, J. Am. Chem. Soc., 2012, 134, 18599-18605.

31 A. Hermann, A. McSorley, N. W. Ashcroft and R. Hoffmann, J. Am. Chem. Soc., 2012, 134, 18606-18618.

32 H. Wang, S. T. John, K. Tanaka, T. Iitaka and Y. Ma, Proc. Natl. Acad. Sci. U. S. A., 2012, 109, 6463-6466.

33 G. Gao, N. Ashcroft and R. Hoffmann, J. Am. Chem. Soc., 2013, 135, 11651-11656.
34 A. D. Becke and K. E. Edgecombe, J. Chem. Phys., 1990, 92, 5397-5403.

35 J. K. Burdett and T. A. McCormick, J. Phys. Chem. A, 1998, 102, 6366-6372.

36 A. B. Migdal, J. Exp. Theor. Phys., 1958, 34, 996.

37 G. M. Eliashberg, J. Exp. Theor.Phys., 1960, 11, 696.

38 P. B. Allen and R. C. Dynes, Phys. Rev. B: Solid State, 1975, 12, 905-922.

39 N. W. Ashcroft, Phys. Rev. Lett., 2004, 92, 187002.

40 G. D. Gaspari and B. L. Gyorffy, Phys. Rev. Lett., 1972, 28, 801805. 